M010

\section{SCREENING PRENATALE PER STREPTOCOCCUS AGALACTIAE: DATI RACCOLTI NEL PERIODO SETTEMBRE 2002 - APRILE 2003}

Genco R., Giannobile G., Puccio G., Turchio B., Verro M., La Chiusa S.

\section{U.O.C. Patologia Clinica, Ospedale Buccheri La Ferla FBF Palermo}

Premessa Lo $S$. agalactiae è responsabile in epoca neonatale di infezioni batteriche a trasmissione verticale potenzialmente mortali. L'infezione precoce può essere trasmessa al neonato per via transplacentare o durante il passaggio attraverso il canale del parto. In relazione all'accumularsi di "evidenze" degli ultimi anni il trattamento antibiotico intrapartum di tutte le gravide colonizzate, sembra essere l'approccio più efficace per la prevenzione delle infezioni precoci da GBS. Scopo dello studio è quindi l'estensione dell'accertamento batteriologico a tutte le gravide che afferiscono ai nostri ambulatori di diagnostica prenatale, la valutazione dei dati riguardanti la percentuale di colonizzazione e lo spettro di resistenza dei ceppi isolati.

Metodi e materiali: il protocollo prevede l'esecuzione del tampone vaginale e rettale in 35-37 settimana di gestazione (il prelievo in ambedue le sedi assicura il maggior valore predittivo di colonizzazione vaginale al momento del parto), suggerisce accurate modalità di prelievo dei campioni batteriologici (sede ottimale per il prelievo è il terzo inferiore della vagina e la parte inferiore del retto) attiva il controllo della batteriuria sintomatica o asintomatica da GBS. Tecniche colturali : arricchimento in brodo Todd-Hewit addizionato con $10 \mu \mathrm{g} / \mathrm{ml}$ di colistina e $15 \mu \mathrm{g} / \mathrm{ml}$ di ac.nalidixico, incubazione 18-24 ore, subcultura in agar sangue montone (Biomerieux) Identificazione presuntiva col CAMP test, tipizzazione sierologia con antisieri specifici (BD), antibiogramma col metodo di microdiluzione in brodo ai breakpoint NCCLS 2002 (Vitek 2).

Risultati Sono state scrinate da settembre 2002 ad aprile 2003, 1028 gravide, sono stati isolati 161 ceppi di GBS, le donne colonizzate sono risultate essere il 15.9\%. Il 100\% dei ceppi isolati sono risultati sensibili ad ampicillina, Penicillina G e vancomicina. Il $20 \%$ ed il $18 \%$ dei ceppi risultano rispettivamente resistenti a clindamicina ed eritromicina. La percentuale di colonizzazione delle gravide del nostro bacino è in linea con i dati della letteratura (dal 10 al $40 \%$ secondo le diverse realtà), piuttosto alta invece la percentuale di ceppi resistenti ad eritromicina e clindamicina dato preoccupante in quanto farmaci di scelta nelle donne con alto rischio di anafilassi per penicillina

Conclusioni: Lo screening batteriologico ha permesso di limitare il trattamento antibiotico alle gravide colonizzate diminuendo il rischio di anafilassi e l'insorgere di nuove resistenze.

\section{M011}

\section{STUDIO POLICENTRICO PER LA VALUTAZIONE DI BCSA,TERRENO SELETTIVO PER BURKHOLDERIA CEPACIA COMPLEX, VERSUS OFPBL}

\author{
Garlaschi ML*, Cariani L. *, Busetti M.**, Grasso E. ^, \\ Grassi P. ^, Belli ML. ${ }^{\wedge}$ e Manno G. ${ }^{\wedge} \wedge$ \\ Garofolo, Trieste; ^ Po *Istituti Clinici di Perfezionamento \\ Milano;*** Burlo licl. Univ., Catania; ^^ Istituto G.Gaslini, Genova
}

Introduzione Burkholderia cepacia Complex, importante patogeno delle vie aeree di pazienti con Fibrosi Cistica (FC), non è di facile isolamento, né di facile identificazione. L'isolamento dall'espettorato di un paziente con FC, di un ceppo di B. cepacia, impone al clinico un comportamento di particolare attenzione verso i pazienti colonizzati, sia terapeutico per la particolare patogenicità, che di segregazione per l'elevata trasmissibilità.

Materiali emetodi Abbiamo condotto, in quattro laboratori di microbiologia (Ist.Clin. Perf., Milano; Ist. G. Gaslini, Genova; IRCCS, Burlo Garofolo, Trieste; Policl. Univ., Catania), uno studio comparativo prospettico per valutare due terreni selettivi per B. cepacia Complex, il BCSA, fornito dalla bioMerieux, Italia, e l' OFPBL, fornito dalla Becton Dickinson Italia. Sono stati processati, dal $15 / 02 / 2002$ al 15/06/2002, 901 campioni provenienti dalle basse vie aeree di 783 pazienti con FC. I due terreni in prova sono stati inseriti nella routine giornaliera. I batteri Gram negativi cresciuti su OFPBL e BCSA sono stati identificati mediante il sistema in uso presso il proprio laboratorio e successivamente riconfermati mediante il metodo API $20 \mathrm{NE}$ bioMerieux. L'identificazione di $B$. cepacia Complex è stata confermata mediante PCR (Agodi JCM 2001 E Bevivino JCM 2002).

Risultati I valori medi, rispettivamente di selettività ed eugonicità dei due terreni valutati, sono stati i seguenti: su BCSA nell' $82.1 \%$ e su OFPBL nell' $11 \%$ di casi non è cresciuto alcun microrganismo, diverso da B. cepacia, ; su BCSA nel $98.4 \%$ e su OFPBL nel 100\% di casi si è evidenziata crescita di B. cepacia.

Discussioni e conclusioni I due terreni selettivi valutati hanno evidenziato una simile eugonicità nei confronti di $B$. cepacia Complex, mentre BCSA nei confronti di OFPBL ha dimostrato una migliore selettività in tutti i Centri di valutazione. Possiamo concludere che l'inserimento di BCSA nella serie di terreni utilizzati per la processazione di materiali respiratori di pazienti con FC è auspicabile e di notevole efficacia.

\section{M012}

\section{RICERCA DI LEGIONELLA E AMEBA DA CAMPIONI DI ACQUA.}

\author{
Franzin L., Cabodi D., Bonfrate N.
}

Sezione Malattie Infettive, Università di Torino, Ospedale "Amedeo di Savoia", Corso Svizzera 164, I 0149 Torino.

Obiettivi: Il genere Legionella comprende batteri acquatici ubiquitari che vivono in simbiosi con le Amebe. La presenza di questi protozoi nell'ambiente risulta importante per il mantenimento e la propagazione del batterio. Le Amebe inoltre svolgono un ruolo rilevante nei meccanismi di virulenza 